\title{
Sistema de Tareas Diagnósticas y la habilidad lectora
}

\section{System of Diagnostic Tasks and relationship reading skill}

\author{
Yaser Ramírez-Benítez ${ }^{1}$ \\ Luis Ernesto Toca-Veliz ${ }^{2}$ \\ Bárbara Bermúdez-Monteagudo ${ }^{3}$ \\ Betsy Martínez-Díaz ${ }^{4}$ \\ Shuyeng Acea Vanega ${ }^{5}$ \\ 1, 2, 3, 4 Universidad de Cienfuegos. Cuba
}

${ }^{5}$ Sala de Rehabilitación del Policlínico "Ramón Claudio Delgado Amestoy”, Cienfuegos. Cuba

\begin{abstract}
Resumen: La presente investigación tiene como objetivo determinar la relación entre el Sistema de Tareas Diagnósticas (STD) y la lectura en una muestra de niños cubanos en el $1^{\circ}$ y $2^{\circ}$ grado. Se aplicaron tres procedimientos: (1) correlaciones entre el STD y la lectura, (2) ANOVA de la Regresión y (3) Regresión jerárquica. Las correlaciones indicaron que las 17 tareas tienen una significativa correlación con la lectura en los dos grados escolares. Sin embargo, el ANOVA de regresión mostró cuatro tareas cognitivas: Análisis fónico, Ordenamiento de láminas, Solución de matrices y Relaciones cuantitativas. En el 2do grado, se destacaron tres tareas: Ordenamiento de láminas, Coordinación viso - motora y Solución de problemas. En el 1er grado, la Regresión jerárquica indicó la importancia de Análisis fónico y el Ordenamiento de láminas por encima de las demás. En el 2do grado, indicó Ordenamiento de láminas y la Coordinación viso - motora. En resumen, las habilidades fonológicas y el razonamiento son procesos básicos que subyacen en la adquisición de la lectura en los primeros grados escolares.
\end{abstract}

Palabras claves: lectura, habilidades fonológicas, preescolar, neurodesarrollo, procesos psicológicos básicos

\begin{abstract}
The objective of this research is to determine the relationship between the System of Diagnostic Tasks (SDT) and the reading in a sample of Cuban children in the 1st and 2nd. Three procedures were applied: (1) correlations between the SDT and the reading, (2) ANOVA of the Regression and (3) Hierarchical regression. The correlations indicated that the 17 tasks have a significant correlation with the reading in the two school grades. However, the regression ANOVA showed four cognitive tasks: Phonic analysis, Sheet ordering, Matrix solution and Quantitative relationships. In the 2 nd grade, three tasks were highlighted: sheet ordering, visuo - motor coordination and problem solving. In the 1st grade, the hierarchical regression indicated the importance of phonic analysis and the ordering of plates above the others. In the 2 nd grade, he indicated sheet ordering and visuo - motor coordination. In summary, phonological skills and reasoning are basic processes that underlie the acquisition of reading in the first grades.
\end{abstract}

Key words: reading, phonological skill, preschool, neurodevelopment, basic psychological process

Cómo citar este artículo:

Ramírez-Benítez, Y., Toca-Veliz, L. E., Bermúdez-Monteagudo, B., Martínez-Díaz, B., \& Acea Vanega, S. (2018). Sistema de Tareas Diagnósticas y la habilidad lectora. Ciencias Psicológicas, 12(1), 79-86. doi: https://doi.org/10.22235/cp.v12i1.1598

Correspondencia: Yaser Ramírez Benítez, Carretera Central, Cuatro Caminos, Cienfuegos, Cuba. Tel. 43549 608, yaser@citmacfg.cu, yramirezbenitez@gmail.com 


\section{Introducción}

El estudio de la lectura es un tema de notable importancia en las edades tempranas por la estrecha relación que guarda con el éxito escolar. En el espacio educativo, la instrucción de la lectura comienza en los primeros grados escolares, debido a que el desarrollo de la habilidad permite el aprendizaje de las nuevas materias a las que se enfrentará el escolar progresivamente (Pinto, Martínez, \& Jiménez, 2016; Aisenberg, 2015).

Los estudios refieren que cuando el niño comienza a recibir instrucción escolar para adquirir la lectura ya cuenta con experiencia cognitiva para aprender la habilidad, básicamente cuenta con destrezas fonológicas, semánticas y ortográficas; procedentes de su experiencia con el lenguaje oral (Fresneda, Mediavilla, \& Pérez, 2017; Valdivieso, 2016). De esta forma, el desarrollo del lenguaje es un prerrequisito importante en los inicios escolares para adquirir la lectura, pues el educador espera condiciones madurativas suficientes en el niño para indicar instrucciones puntuales y sistemáticas. No obstante, algunos estudios muestran que el lenguaje no es el único factor a considerar (Arán-Filippetti \& López, 2016; Ferroni, Diuk, \& Mena, 2016).

En este sentido, algunos estudios han reportado la importancia que tiene la memoria de trabajo y la velocidad de procesamiento, como predictores de la lectura de palabras (Kim \& Pallante, 2012; Ziegler et al., 2010). En iguales condiciones, la inteligencia es otra función que explica el rendimiento lector en la etapa escolar, tanto la inteligencia fluida (Gf o capacidad para resolver problemas sin experiencia y aprendizaje previo) como la inteligencia cristalizada (Gc o riqueza, amplitud y profundidad del conocimiento adquirido) (Ritchie, Bates, \& Plomin, 2015; Song et al., 2015).

Desde esta perspectiva, los investigadores han llegado a un consenso sobre las condiciones previas que debe tener el niño preescolar para adquirir la lectura, básicamente se destacan la importancia de componentes cognitivos generales (la inteligencia, la atención, la memoria) y específicos (lenguaje) para desarrollar la habilidad (Cuetos et al., 2015).

Dentro de los modelos de desarrollo de la lectura, se destaca la teoría de doble ruta de Ellis y Young (1988), la cual ha sido justificada por varias evidencias empíricas (Mosquera, 2012;
Coltheart, 2006). Los autores asumen la existencia de dos vías para el procesamiento de la palabra escrita: la directa e indirecta. La vía directa (ruta léxica o semántica), asocia la forma ortográfica de la palabra con su significado interno, mientras que en la vía indirecta (ruta fonológica) se relacionan los grafemas de la palabra con sus sonidos correspondientes y se accede al significado de esta por medio del sonido de los grafemas que la conforman.

A pesar que ambas vías tienen una fuerte influencia sobre la adquisición de la lectura, algunos autores reconocen a la ruta fonológica como la más importante para pronosticar el desarrollo de la lectura. El fuerte efecto de la ruta fonológica es justificada por varias evidencias empíricas: estudios sobre dislexia (Neuhoff et al., 2012; Gabor et al., 2011), estudios sobre intervención para entrenar la lectura (Ferroni, Mena, \& Diuk, 2016) y estudio longitudinales sobre el desarrollo de la lectura (Suárez-Coalla, García-de-Castro, \& Cuetos, 2013). Asimismo, otros investigadores muestran que la contribución de ambas rutas están influenciadas por las características del idioma (Suárez-Coalla et al., 2013).

En este sentido, los estudios transculturales abordan la importancia de la ortografía del idioma, pues tiene una influencia notable en la velocidad de adquisición del código lector y en la prontitud para alcanzar los grados de lectura experta. Algunos estudios reportan que los factores fonológicos serían importantes predictores de lectura en cualquier idioma (Hudson et al., 2013; Sellés, et al., 2012; Ziegler et al., 2010; Smith, 2012), aunque al parecer las habilidades fonológicas tienen un pico de desarrollo más temprano en los idiomas transparentes, puesto que a los nueve años la estrategia semántica contribuye mejor a pronosticar la eficiencias lectora que la ruta fonológica (Mosquera, 2012). De esta forma, adquirir la lectura en idiomas opacos (inglés, francés) es más difícil que en idiomas transparentes (español, alemán).

En iguales condiciones, el método de enseñanza de la lectura es un punto a considerar en el análisis, pues la manera que se instruye la lectura tiene un efecto duradero sobre el rendimiento de los niños. De esta forma, según el método que se emplee, algunos factores madurativos adquirirán mayor relevancia que otros. Asimismo, Jiménez y Rumeu (1989), muestran, en un estudio longitudinal, el efecto de los métodos globales y sinté- 
ticos en una población escolar de habla hispana. Los autores reportan que los niños que aprendieron a través del método sintético presentaron dificultades en la compresión de textos, mientras que los niños que aprendían de un modo global tenían más errores en la codificación fonema-grafema. Iguales resultados fueron encontrados por otros investigadores (Jiménez \& Guzmán, 2003), los cuales reportaron un efecto de superioridad en el análisis fonológico en niños que aprendían a leer a través del método sintético, sin embargo, los niños que aprendían a leer por el método global experimentaron mayores dificultades en el nombrado de palabras cuando intervenían procesos de mediación fonológica. Por esta razón, y considerando las premisas de la instrucción escolar de la lectura, en Cuba se utilizan los dos métodos para enseñar la lectoescritura, pues la exigencia recae no solo en la separación fonológica de la palabra, también en su significado, de manera tal que los procesos sean paralelos.

En Cuba, la enseñanza de la lectura se realiza paralela a la escritura, considerando que ambas van a la par. Desde esta perspectiva, el sistema educacional cubano establece como objetivo general: Desarrollar las habilidades en los alumnos que le permitan leer y comprender textos breves y de diferente género y expresar por escrito, en sencillas oraciones, sus ideas y experiencias (Díaz González, 2017).

En el primer grado escolar, la enseñanza cubana divide su instrucción en dos partes, la primera en la etapa de aprestamiento cuyo propósito es crear las condiciones necesarias para un buen aprendizaje y otra etapa de enseñanza. La organización de la etapa de aprestamiento está en función de las condiciones reales del grupo y del niño en particular, el cual es realizado a través del diagnóstico preescolar con el instrumento educativo Sistema de Tarea Diagnósticas (STD). En esta etapa, el STD es importante para los educadores, pues a través de este y otras técnicas cualitativas se diseña una estrategia educativa en el aula, en especial en los niños de 1 er grado.

El STD es una alternativa psicométrica para conocer el nivel de desarrollo en la Educación Preescolar, así como hacer valoraciones para pronosticar las habilidades escolares básicas en el 1er y 2do grado académico (López \& Silverio, 1996). En los últimos diez años, la Educación Preescolar en Cuba ha utilizado el STD como instrumento efectivo para trazarse estrategias educativas en esta enseñanza. Básicamente, los reportes anuales indican al lenguaje oral como la habilidad con mayores dificultades para adquirir en esta etapa de la vida. No obstante, otras investigaciones, destacan otros problemas en la infancia preescolar, tales como en el razonamiento verbal, en la inhibición conductual, en la motricidad manual y en la organización espacial (Ramírez Benitez, Jiménez-Morales., Díaz Bringas, \& Fárdales Macías, 2015.; Ramírez \& Jiménez-Morales, 2014).

Desde esta perspectiva, el niño preescolar cubano no solo presenta problemas en el lenguaje, también en otras habilidades mentales superiores que comprometen la adquisición de las habilidades escolares básicas.

En este sentido, el Departamento de Logopedia, en la Universidad de Cienfuegos y el Instituto de Neurociencias de la Universidad Favaloro, Argentina, desarrollan un grupo de investigaciones encaminadas a relacionar el diagnóstico preescolar con el rendimiento académico en los primeros grados escolares (1 ero y 2 do grado) y con el objetivo de destacar las principales habilidades superiores que contribuyan a la adquisición de las habilidades escolares básicas como la lectoescritura y la matemática. La línea de investigación es importante, pues indica los principales componentes del desarrollo para estimular las habilidades escolares desde la etapa preescolar, ya sea en niños con desarrollo típico para su edad o en poblaciones con necesidades educativas especiales. Además, en la literatura no se reportan estudios donde se relacionen los valores alcanzados en el test STD con el aprendizaje escolar.

Considerando estos argumentos y la importancia de relacionar el nivel de desarrollo alcanzado por el niño con la habilidad lectora, la presente investigación pretende realizar un estudio longitudinal con el objetivo de determinar la influencia del STD sobre la habilidad lectora en el $1^{\circ}$ y $2^{\circ}$ grado escolar. El instrumento STD se aplica en niños de cinco y seis años, sin embargo la investigación pretende obtener las puntuaciones del niño a los 5 años y después valorar esas puntuaciones con el rendimiento de la lectura un años después en el 1er grado, y dos años después en el 2 do grado.

Se espera, que el desarrollo del lenguaje tenga significativa influencia en el desarrollo de la habilidad lectora, tal y como reporta la literatura. 


\section{Método}

\section{Participantes}

La investigación se realizó en una institución educativa en la provincia de Cienfuegos, Cuba. Se utilizó una muestra no probabilística de tipo intencional en la institución educativa, pues esta institución es parte del proyecto Educación inclusiva de esa institución. Se consideraron algunos criterios de selección: a) niños que cursan el 1ero y 2do grado de la escuela regular Antonio Maceo desde el inicio hasta el final de curso. b) tener todas las notas actualizadas en el expediente preescolar. c) presentar el consentimiento de los padres. De 155 niños, participaron 143 escolares: 68 de primer grado ( 35 féminas y 33 varones) y 75 niños en 2 do grado (39 féminas y 36 varones). Quedaron excluidos 12 niños por no presentar notas del STD en su expediente académico.

\section{Descripción del instrumento}

- Sistema de Tareas Diagnósticas (STD, López \& Silverio, 1996): Es un instrumento educativo para evaluar el desarrollo del niño preescolar. Está conformado por 17 tareas cognitivas en cuatro áreas o dominios: Lenguaje, Motricidad, Percepción y Establecimiento de relaciones. La calificación se realiza en cada tarea a través de 5 indicadores: 5 puntos (excelente), 4 puntos (bueno), 3 puntos (Regular), 2 (Mal) y 1 (Deficiente). De manera general, se puede calificar de 4 formas: Perfil con Meseta Alta (con puntuaciones entre 4 y 5 puntos en las 17 tareas), Perfil con Meseta Media (con puntuaciones de 3 en las diferentes pruebas), Perfil de Meseta Baja (con puntuaciones entre 1 y 2 puntos) y el Perfil Inarmónico (muestra grandes variaciones entre las puntuaciones de las 17 tareas). Las 17 tareas se agrupan 4 Áreas: (1) Lenguaje: Análisis fónico (AF), Pronunciación (P) y Lenguaje relacional (LR). (2) Motricidad fina: Coordinación viso-motora (CVM), Trazado de rasgos (TR), Rasgado (R), Recorte (RE), Coloreado (C) y Trazado (T). (3) Percepción visual: Forma (F), Semejanzas y diferencias (SD), Organización perceptual (OP) y Fondo - Figura (FF). (4) Establecimiento de relaciones: Ordenamiento de láminas $(\mathrm{OL})$, Relaciones cuantitativas $(\mathrm{RC})$, Solución de problemas (SP) y Solución de matrices (SM).

- Corte evaluativo: Resultado emitido por la maestra en las asignaturas básicas (lectura) des- pués de tres meses de instrucción escolar (corte evaluativo inicial) y a finales de la etapa escolar (corte evaluativo final).

\section{Procedimiento}

En el expediente académico se seleccionaron dos puntuaciones: (1) resultados en las 17 tareas obtenidas por los niños a los 5 años (Variables independientes), tanto en el grupo de niños de 1er grado como en el grupo de 2 do grado, (2) resultados del rendimiento en la lectura en los inicios y finales del grado, tanto en el grupo de niños de 1er grado como en el grupo de 2 do grado (Variables dependiente).

\section{Procedimiento estadístico}

- Coeficiente de Pearson con el objetivo de conocer las correlaciones entre las variables del STD y el rendimiento de la lectura en el corte evaluativo inicial y en corte evaluativo final.

- ANOVA de regresión con el objetivo de destacar las tareas del STD con mayor peso predictor. Variable dependiente (rendimiento en la lectura: corte evaluativo inicial y corte evaluativo a finales del curso) y variables independientes (17 tareas del STD).

- Regresión jerárquica con el objetivo de comprobar los resultados obtenidos en el ANOVA de regresión. Variable dependiente (rendimiento en la lectura: corte evaluativo final) y variables independientes (tareas del STD con valores significativos en el ANOVA de regresión, y al mismo tiempo, que hayan mantenido su efecto significativo en los dos momentos de la evaluación: corte evaluativo inicial y final).

\section{Resultados}

\section{Análisis de correlaciones}

El análisis de correlación indicó correlaciones significativas entre las 17 tareas del STD y el rendimiento lector, tanto con corte evaluativo de ler grado como en el corte evaluativo de 2 do grado. De manera general, las correlaciones entre las variables fueron disminuyendo a medida que aumentaba el grado escolar.

En el 1ero grado, las correlaciones entre el STD y el corte evaluativo inicial (3 meses iniciado el curso) fueron significativas, con valores 
entre 0,50 y 0,$66 ; p \leq, 01$. En igual sentido, las correlaciones entre el STD y el corte evaluativo final fueron positivas con valores entre 0,35 y 0,$57 ; p \leq$ ,01 (ver tabla 1).

Tabla 1

Análisis de correlación entre el STD y el corte evaluativo inicial y final de la lectura en el 1ero y 2do grado

\begin{tabular}{|c|c|c|c|c|}
\hline & \multicolumn{2}{|c|}{ 1ero grado } & \multicolumn{2}{|c|}{2 do grado } \\
\hline & $\begin{array}{l}\text { Lectura } \\
\text { (corte } \\
\text { inicial) }\end{array}$ & $\begin{array}{l}\text { Lectura } \\
\text { (corte } \\
\text { final) }\end{array}$ & $\begin{array}{l}\text { Lectura } \\
\text { (corte } \\
\text { inicial) }\end{array}$ & $\begin{array}{l}\text { Lectura } \\
\text { (corte } \\
\text { final) }\end{array}$ \\
\hline $\mathrm{AF}$ & $.609(* *)$ & $.541(* *)$ & $.325(* *)$ & $.308(* *)$ \\
\hline$P$ & $.607(* *)$ & $.488(* *)$ & .183 & .159 \\
\hline LR & $.574(* *)$ & $.518(* *)$ & $.343(* *)$ & $.375(* *)$ \\
\hline CVM & $.665(* *)$ & $.428(* *)$ & $.405(* *)$ & $.402(* *)$ \\
\hline TR & $.495(* *)$ & $.350(* *)$ & $.302(* *)$ & $.270(*)$ \\
\hline RA & $.610(* *)$ & $.526(* *)$ & $.246(*)$ & $.295(* *)$ \\
\hline $\mathrm{RE}$ & $.618(* *)$ & $.533(* *)$ & $.264(*)$ & .181 \\
\hline $\mathrm{C}$ & $.512(* *)$ & $.377(* *)$ & $.390(* *)$ & $.347(* *)$ \\
\hline $\mathrm{T}$ & $.592(* *)$ & $.473(* *)$ & $.314(* *)$ & $.269(*)$ \\
\hline $\mathrm{F}$ & $.509(* *)$ & $.396(* *)$ & $.410(* *)$ & $.321(* *)$ \\
\hline SD & $.543(* *)$ & $.528(* *)$ & $.299(* *)$ & $.274(*)$ \\
\hline OP & $.620(* *)$ & $.533(* *)$ & $.273(*)$ & $.294(* *)$ \\
\hline $\mathrm{FF}$ & $.548(* *)$ & $.492(* *)$ & $.341(* *)$ & $.345(* *)$ \\
\hline OL & $.629(* *)$ & $.556(* *)$ & $.417(* *)$ & $.473(* *)$ \\
\hline $\mathrm{RC}$ & $.610(* *)$ & $.573(* *)$ & .146 & $.277(*)$ \\
\hline SP & $.589(* *)$ & $.567(* *)$ & $.350(* *)$ & $.273(*)$ \\
\hline \multirow[t]{2}{*}{ SM } & $.661(* *)$ & $.568(* *)$ & $.308(* *)$ & $.365(* *)$ \\
\hline & 75 & 75 & 68 & 68 \\
\hline
\end{tabular}

**La correlación es significante al nivel 0,01 (unilateral)

*La correlación es significativa al nivel 0,05 (unilateral)

El análisis en el 2do grado, también mostró correlaciones significativas, aunque menores comparado con la muestra de niños de 1er grado. En el corte evaluativo inicial, de 17 tareas solo diez mostraron correlaciones significativas con valores entre 0,29 y 0,$41 ; p \leq, 01$. Las tareas de RE, RA, $\mathrm{RC}$, OP, y P no mostraron correlaciones significativas. En el corte evaluativo final, de 17 tareas solo ocho mostraron correlaciones significativas con valores entre 0,30 y 0,$47 ; p \leq, 01$. Las tareas de $\mathrm{P}$, TR, RE, T, SD, RC, SP no mostraron correlaciones significativas (ver tabla 1).

\section{ANOVA de regresión}

El ANOVA de regresión indicó que en el 1er grado, ocho tareas de 17, mostraron valores signi- ficativos para pronosticar el rendimiento de la lectura en los primeros tres meses del curso escolar: SM, CVM, OP, OL, R, RC, AF y P. Este mismo análisis a finales del curso, indicó que las tareas con valores significativos para pronosticar fueron: RC, SM, SP, OL y AF (ver tabla 2). Finalmente, las tareas que mostraron valores significativos en los dos momentos del corte evaluativo fueron: SM, AF, OL y RC.

En el 2do grado, el ANOVA de regresión mostró cinco tareas de 17 con valores significativos para pronosticar el rendimiento lector en los primeros tres meses: OL, F, CVM, C y SP. Mientras que a finales de la evaluación del 2 do grado, se destacaron solo 4 tareas: OL, CVM, LR y SM (ver tabla 2). En resumen, las tareas que mostraron valores significativos en los dos momentos del corte evaluativo fueron: OL, CVM y SM.

Tabla 2

ANOVA de regresión entre las tareas cognitivas (STD) y el rendimiento lector en dos momentos: corte evaluativo inicial y corte evaluativo final de curso

\begin{tabular}{|c|c|c|c|c|c|c|}
\hline \multicolumn{7}{|c|}{ 1 er grado } \\
\hline \multirow[b]{2}{*}{ Tareas } & \multicolumn{3}{|c|}{$\begin{array}{l}\text { Corte evaluativo } \\
\text { (Primeros } 3 \text { meses del } \\
\text { curso) }\end{array}$} & \multicolumn{3}{|c|}{$\begin{array}{l}\text { Corte evaluativo } \\
\text { (Evaluación final del } \\
\text { curso) }\end{array}$} \\
\hline & $R 2$ & $F$ & $p$ & $R 2$ & $F$ & $p$ \\
\hline $\mathrm{AF}$ & .49 & 23.488 & .000 & .33 & 8.861 & ,000 \\
\hline $\mathrm{P}$ & .54 & 28.103 & .000 & - & - & - \\
\hline CVM & .56 & 30.185 & .000 & - & - & - \\
\hline $\mathrm{RE}$ & .76 & 78.114 & .000 & - & - & - \\
\hline $\mathrm{OP}$ & .76 & 79.100 & .000 & - & - & - \\
\hline $\mathrm{OL}$ & .67 & 49.597 & .000 & .43 & 13.554 & .000 \\
\hline $\mathrm{RC}$ & .72 & 62.095 & .000 & .48 & 16.475 & .000 \\
\hline SM & .58 & 32.882 & .000 & .39 & 11.262 & .000 \\
\hline SP & - & - & - & .39 & 11.650 & .000 \\
\hline \multicolumn{7}{|c|}{ 2do grado } \\
\hline & \multicolumn{3}{|c|}{$\begin{array}{l}\text { Corte evaluativo } \\
\text { (Primeros } 3 \text { meses } \\
\text { del curso) }\end{array}$} & \multicolumn{3}{|c|}{$\begin{array}{l}\text { Corte evaluativo } \\
\text { (Evaluación final } \\
\text { del curso) }\end{array}$} \\
\hline Tareas & $R 2$ & F & $p$ & $R 2$ & F & $p$ \\
\hline LR & - & - & - & 0.34 & 8.250 & .000 \\
\hline CVM & .32 & 7.748 & .000 & 0.35 & 8.541 & .000 \\
\hline $\mathrm{C}$ & .26 & 5.823 & .000 & - & - & - \\
\hline $\mathrm{F}$ & .23 & 4.908 & .001 & - & - & - \\
\hline $\mathrm{OL}$ & .20 & 4.172 & .004 & 0.40 & 10.856 & .000 \\
\hline SP & .17 & 3.408 & .01 & - & - & - \\
\hline SM & - & - & - & 0.27 & 6.016 & .000 \\
\hline
\end{tabular}




\section{Análisis de regresión jerárquica}

En el 1er grado, el análisis de regresión jerárquica indicó que el Análisis fónico $(\mathrm{AF})$ y el Ordenamiento de láminas $(\mathrm{OL})$ son predictores significativos de la habilidad lectora.

El AF explica el 37\% ( $\mathrm{F}=43,096 ; p<, 001)$ de la varianza del rendimiento lector cuando se controla el efecto de OL en los primeros 3 meses del curso. En iguales condiciones, OL explica el $44 \%(\mathrm{~F}=9,760 ; p<, 01)$ de la varianza del rendimiento lector cuando se controla el efecto de $\mathrm{AF}$ (ver tabla 3). Las demás variables (SM y RC) no mostraron contribución significativa.

A finales del curso, AF explica el 29\% $(\mathrm{F}=30,204 ; p<, 001)$ de la varianza del rendimiento lector. Mientras que OL explica el 35\% $(\mathrm{F}=6,382 ; p<0,5)$ del rendimiento lector.

En el 2do grado, el análisis de regresión jerárquica indicó que las tareas de Coordinación viso - motora (CVM) y el Ordenamiento de láminas (OL) contribuyen de manera significativa en

Tabla 3

Regresión jerárquica entre las tareas cognitivas seleccionadas en el ANOVA de regresión y el rendimiento lector

\begin{tabular}{|c|c|c|c|}
\hline \multicolumn{4}{|c|}{ 1er grado } \\
\hline & \multicolumn{3}{|c|}{$\begin{array}{c}\text { Corte evaluativo } \\
\text { (Primeros } 3 \text { meses del curso) }\end{array}$} \\
\hline Modelo1 & $R 2$ & Cambios en $F$ & $p$ \\
\hline $\mathrm{AF}$ & 0.37 & 43,096 & .000 \\
\hline $\mathrm{AF}, \mathrm{OL}$ & 0.44 & 9,760 & .003 \\
\hline $\mathrm{AF}, \mathrm{OL}, \mathrm{RC}$ & 0.45 & 0,450 & .505 \\
\hline $\mathrm{AF}, \mathrm{OL}, \mathrm{SM}$ & 0.47 & 4,395 & .040 \\
\hline Modelo 2 & \multicolumn{3}{|c|}{$\begin{array}{c}\text { Corte evaluativo } \\
\text { (Evaluación final del curso) }\end{array}$} \\
\hline $\mathrm{AF}$ & .293 & 30.204 & .000 \\
\hline $\mathrm{AF}, \mathrm{OL}$ & .350 & 6.382 & .014 \\
\hline $\mathrm{AF}, \mathrm{OL}, \mathrm{RC}$ & .365 & 1.681 & .199 \\
\hline $\mathrm{AF}, \mathrm{OL}, \mathrm{SM}$ & .377 & 1.354 & .248 \\
\hline \multicolumn{4}{|c|}{ 2do Grado } \\
\hline & \multicolumn{3}{|c|}{$\begin{array}{c}\text { Corte evaluativo } \\
\text { (Primeros } 3 \text { meses del curso) }\end{array}$} \\
\hline Modelo 3 & $R 2$ & Cambios en $F$ & $p$ \\
\hline CVM & .16 & 12.946 & .001 \\
\hline CVM, OL & .22 & 4.852 & .031 \\
\hline Modelo 4 & \multicolumn{3}{|c|}{$\begin{array}{c}\text { Corte evaluativo } \\
\text { (Evaluación final del curso) }\end{array}$} \\
\hline CVM & .16 & 12.718 & .001 \\
\hline CVM, OL & .25 & 8.312 & .005 \\
\hline
\end{tabular}

el rendimiento de la lectura, tanto al principio del grado como a finales de la etapa.

En los primeros 3 meses del curso, la tarea CVM explica el 16\% ( $\mathrm{F}=12,946 ; \quad p<, 01)$ de la varianza del rendimiento lector. En iguales condiciones, OP explica el 22\% ( $\mathrm{F}=4,852 ; p<, 05)$, de la varianza del rendimiento lector (Véase la tabla 3 ).

A finales del curso escolar, CVM explica el $16 \%(\mathrm{~F}=12,718 ; p \leq, 001<, 01)$ de la varianza del rendimiento lector, mientras que OP explica el $25 \%(\mathrm{~F}=8,312 ; p \leq, 005<, 01)$.

\section{Discusión}

Los resultados obtenidos en la presente investigación indicaron que el desarrollo alcanzado por el niño a los 5 años es un indicador importante para pronosticar la habilidad lectora en el $1^{\circ}$ y $2^{\circ}$ grado escolar. De esta manera, el desarrollo del lenguaje no es el único factor a considerar en la infancia preescolar para explicar el futuro desempeño de la lectura.

Según el análisis de correlaciones, todas las áreas del desarrollo son importantes para explicar la lectura en el 1er y 2do grado escolar. Sin embargo, el ANOVA de regresión indicó que algunas tienen mayor peso para pronosticar.

En el primer grado la influencia del AF es notable y significativa, tanto en los inicios del aprendizaje como a finales del grado. El resultado es consistente con varias investigaciones (AránFilippetti \& López, 2016; Ferroni, Mena, \& Diuk, 2016; Ramírez \& Jiménez, 2014;. González Seijas et al., 2013; Escoba \& Meneses, 2014; SuárezCoalla, García-de-Castro, \& Cuetos, 2013; Sellés, Martínez \& Vidal-Abarca, 2012), puesto que el niño necesita del AF para avanzar en la adquisición, además el método de enseñanza que se realiza en nuestro contexto (analítico - fónico) posibilita que la habilidad fonológica sea relevante en los inicios de la adquisición lectora.

No obstante, el AF no fue la única variable relevante en el 1er grado. Se destacan otras tareas como SM, el OL y las RC, lo cual indica la importancia de otras medidas relacionadas con el razonamiento. Sin embargo, un análisis estadístico más rigoroso, como la regresión jerárquica, reportó que OL y AF tuvieron un mayor peso para explicar la lectura. Es decir, las tareas que miden el área del pensamiento son importantes predictoras de la habilidad lectora, aunque OL tuvo un mayor 
peso para explicarla. Mientras que en el área del lenguaje AF explicó con mayor varianza.

Este resultado final corrobora el uso paralelo de ambas estrategias (fonológica y semántica) en el desarrollo de la lectura. En este sentido, en la medida que se aprende a coordinar el fonema con el grafema, se comprende el texto, lo cual demanda de procesos cognitivos generales que tributan a la estrategia semántica, esencialmente tareas relacionadas con el pensamiento visual. Este argumento resulta interesante, pues los investigadores, generalmente, reconocen a la estrategia semántica como un componente del lenguaje y valoran esta función desde una tarea de vocabulario, sin embargo, las tareas del pensamiento tributan al desarrollo de dicha estrategia, esencialmente para comprender el texto con funciones básicas como el análisis, la síntesis y la generalización de ideas. Es por ello, que algunos investigadores destacan la importancia de la inteligencia para pronosticar la lectura, y en especial, la inteligencia fluida, pues contribuye de manera general en la compresión de texto, ya sea en contexto de habla inglesa (Ritchie, Bates, \& Plomin, 2015; Song et al., 2015) como de habla hispana (Ramírez, Torres, \& Amor, 2016; 2017).

En el segundo grado, el AF no pierde valor pronóstico, aunque los valores van decayendo comparado con otras tareas de tipo semántico. En otras palabras, resulta relevante la estrategia semántica y otras habilidades cognitivas generales que tributan al desarrollo léxico, tales como el OL, la SP y la CVM.

De esta forma, el niño está más enfocado en comprender más el texto que en aprender los sonidos de la escritura. Además en el 2 do grado, el método de enseñanza sigue siendo el mismo (fónico - analítico), aunque con mayor énfasis en la compresión y la escritura, ya que el niño ha ido automatizando algunos sonidos con el grafema. Es por ello, que la CVM se destaca como variables significativas para pronosticar, pues en la medida que el niño lee y comprende es capaz de focalizar la grafía del sonido, o al menos se le exige que lo haga. De esta manera, se entrena la coordinación de habilidades complejas entre la motricidad y la percepción, la cual es indicador de desarrollo de regiones cerebrales asociativas y su conectividad.

Anteriormente, en el 1er grado, el niño estaba orientado a relacionar el sonido con el grafema y a comprender el texto, mientras en 2 do grado orienta su esfuerzo para comprender y escribir el sonido. Al parecer la variable fonológica pierde capacidad predictiva para pronosticar la habilidad lectora en el 2do grado escolar, lo cual es consistente con otros autores cuando realizan el análisis en niños alemanes en el 2do grado (Wimmer \& Schurz, 2010) y en niños cubanos en el tercer grado (Mosquera, 2012). Sucede así, ya que tanto el alemán como el español son idiomas transparentes y las destrezas fonologías se adquieren más tempranas en el desarrollo y el esfuerzo del niño se centra en habilidades de compresión.

No obstante, las bajas puntuaciones en el análisis fónico, tanto en el niño de 1 ero como de 2 do grado, es objeto de alerta en los educadores, pues se recomiendarealizaracciones educativas diferenciales en estos niños, tanto en los primeros tres meses iniciales del curso escolar como a finales del curso.

De manera general, los resultados aportados por el presente estudio, respecto a la relación entre el STD y el rendimiento de la lectura en los primeros años escolares, tienen implicaciones para la práctica educativa, esencialmente en la educación preescolar. La primera está relacionada con la capacidad predictiva de la tarea AF sobre la habilidad lectora en los niños de 1ero grado, así se podría detectar niños con bajos resultados a los 5 años o a los inicios del grado y organizar tareas de estimulación que compensen las dificultades. Igualmente, los educadores de 2 do grado debe estar alerta al diagnóstico del STD en los inicios del grado, pues el niño con bajos resultados a los cinco o seis años, podría mantener las dificultades fonológicas en la primera etapa del grado.

La segunda implicación práctica está relacionada con las tareas de pensamiento y motricidad del STD, pues en la infancia preescolar se estimula con mucha frecuencia las tareas de motricidad por existir varios juegos didácticos relacionados con esta habilidad y en menor medida se trabaja con el razonamiento, pues son juegos que requieren menos movimientos y suele ser aburrido para los niños. De esta forma, se considera importante la evaluación y la estimulación del razonamiento en la infancia preescolar, pues guarda significativa relación con el desarrollo inicial de la lectura.

Igualmente, los educadores deben considerar las bajas puntuaciones en la tarea de CVM, tanto a los cinco años como a los seis años, pues refleja el estado madurativo de las regiones cerebrales asociativas, esencialmente la mielinización entre las regiones frontales y parietales. La coordinación mano - ojo, fonema - grafema y pensamiento lenguaje, son procesos básicos para adquirir la lec- 
toescritura, los cuales podría ser valorados desde las puntuaciones de la tarea CVM.

A modo de conclusión, la presente investigación destaca la importancia que tiene el desarrollo del lenguaje, el pensamiento y la motricidad en la infancia preescolar para establecer pronósticos de la lectura en los primeros grados escolar. De esta forma, el nivel de desarrollo alcanzado en la infancia preescolar es un indicador importante para pronosticar el futuro desempeño académico del niño, en especial la habilidad lectora.

\section{Referencias}

Aisenberg, B. (2015). Aprender Historia en prácticas de lectura compartida. LiDEs-A literacia das disciplinas escolares: Desafios nas aulas de História e Matemática, 55.

Arán-Filippetti, V., \& López, M.B. (2016). Predictores de la Comprensión Lectora en niños y adolescentes: El papel de la edad, el sexo y las funciones ejecutivas. Cuadernos de Neuropsicología, 10(1), 23-44. doi: 10.7714/CNPS/10.1.202

Coltheart, M. (2006). Dual route and connectionist models of reading: on overview. London Review of Education, 4(1), 5-17. doi: 10.1080/13603110600574322

Cuetos, F., Súarez Coalla, P., Molina, MI., \& Llenderrozas, MC. (2015). Test de detección temprana de las dificultades en el aprendizaje de la lectura y escritura. Pediatría Atención Primaria, 17(66), e99-e107. http://dx.doi.org/10.4321/ S1139-76322015000300002.

Díaz González, M. (2017). Concepción de la educación de la primera infancia cubana. La Habana: Educación Cubana.

Ellis, A.W. \& Young, A.W. (1988). Human Cognitive Neuropsychology. Hove, UK: Erlbaum.

Escobar, J.P., \& Meneses, A. (2014). Predictores de la lectura inicial en español según NSE: ¿es suficiente la semi-transparencia para explicar su desempeño? Estudios de Psicología, 35(3), 625-635.

Ferroni, M.V., Diuk, B., \& Mena, M. (2016). Desarrollo de la lectura y la escritura de palabras con ortografía compleja: sus predictores. Avances en Psicología Latinoamericana, 34(2), 253-271. doi: 10.7714/CNPS/10.1.202

Ferroni, M.V., Mena, M., \& Diuk B. (2016). Niveles de respuestas a una intervención en ortografia. Ciencias Psicológicas, 10(1), 55-61.

Fresneda, R. G., Mediavilla, A. D., \& Pérez, E. J. (2017). Estudio longitudinal sobre el aprendizaje lector en las primeras edades. Revista de educación, (378), 30-51. doi:10.4438/1988592X-RE-2017-378-360

Gabor, S., Fosker, T., Huss, M., Mead, N., Szucs, D., \& Goswami, U. (2011). Auditory sensory deficits in developmental dyslexia: A longitudinal ERP study. NeuroImage, 57(3), 723732. doi: 10.1016/j.neuroimage.2011.04.005

González Seijas, R.M., López Larrosa, S., Vilar Fernández, J., \& López-Vázquez, A. (2013). Estudio de los predictores de la lectura. Revista de Investigación en Educación, 11(2), 98-110.

Hudson Pérez, M.C., Förster Marín, C.E., Rojas-Barahona, CA., Valenzuela Hasenohr, MF., Riesco Valdés, P., \& Ramaciotti Ferré, A. (2013). Comparación de la efectividad de dos estrategias metodológicas de enseñanza en el desarrollo de la comprensión lectora en el primer año escolar. Perfiles Educativos, XXXV (140), 100 - 118.

López, H., \& Silverio, G. (1996). El diagnóstico: un instrumento de trabajo pedagógico de preescolar a escolar. La Habana: Editorial Pueblo y Educación.

Jiménez, J.E., \& Rumeu, M. (1989). Writing disorders and their relationship to reading-writing methods: A longitudinal study. Journal of Learning Disabilities, 22, 195-199.
Jiménez, J.E., \& Guzmán, R. (2003). The influence of codeoriented versus meaning-oriented approaches to reading instruction on word recognition in the Spanish language. International Journal of Psychology, 38, 65-78.

Kim, Y. S., \& Pallante, D. (2012). Predictors of reading skills for kindergartners and first grade students in Spanish: a longitudinal study. Reading and Writing, 25 (1), 1 - 22. doi: 10.1007/s11145-010-9244-0

Mosquera R. (2012). Valor predictivo de la lectura de palabras en la competencia lectora en el idioma español. Un estudio longitudinal. (Tesis de Maestría en Neurociencias). Centro de Neurociencias de Cuba. Habana, Cuba. Disponible en la Biblioteca del Centro de Neurociencias de Cuba, www. cneuro.edu.cu

Neuhoff, N., Bruder, J., Bartling, J., Warnke, A., Remschmidt, H., Müller-Myhsok, B., \& Schulte-Körne, G. (2012). Evidence for the Late MMN as a Neurophysiological Endophenotype for Dyslexia. PLoS ONE, 7 (5):e34909. doi:10.1371/journal.pone.0034909

Pinto, N. S., Martínez, A. I. M., \& Jiménez-Taracido, L. (2016). Learning strategies, reading comprehension and academic achievement in Secondary Education. Psicologia Escolar e Educacional, 20(3), 447-456. http://dx.doi. org/10.1590/2175-353920170211950

Ritchie, S.J., Bates, T.C., \& Plomin, R. (2015). Does Learning to Read Improve Intelligence? A longitudinal multivariate analysis in identical twins from age 7 to 16 . Child Development, 86(1), 23-36. doi: 10.1111/cdev.12272

Ramírez Benítez, Y., \& Jiménez Morales, RM. (2014). Aplicación de la prueba Luria Inicial en el niño preescolar con desviaciones del neurodesarrollo en Cuba. Gaceta Médica Espirituana, 16(3), 1 - 9.

Ramírez Benítez, Y., Jiménez-Morales, R.M., Díaz Bringas, M., \& Fárdales Macías, V.E. (2015). Normas cubanas del instrumento neuropsicológico Luria Inicial para niños preescolares 4 - 6 años. Cuadernos de Neuropsicología, 9(2), 49-69.

Ramírez Benítez, Y., Torres Díaz, R., \& Amor Dias, V. (2017). Batería de Rendimiento Intelectual para niños escolares cubanos. Rev. Chil. Neuropsicol. 12(2), 20-27. doi: 10.5839/ renp.2017.12.02.04

Ramírez Benítez, Y., Torres Díaz, R., \& Amor Dias, V. (2016). Contribución única de la inteligencia fluida y cristalizada en el rendimiento académico. Rev. Chil. Neuropsicol, 11(2), 1-5. doi: 10.5839/renp.2016.11.02.01

Song, S., Su, M., Kang, C., Liu, H., Zhang, Y., McBride-Chang, C., ... Shu, H. (2015). Tracing children's vocabulary development from preschool through the school-age years: An 8-year longitudinal study. Development Science, 18(1), 119-131. doi: 10.1111/desc.12190

Suárez-Coalla, P., García-de-Castro, M., \& Cuetos, F. (2013). Variables predictoras de la lectura y la escritura en castellano. Infancia y Aprendizaje, 36(1), 77-89. doi:10.1174/021037013804826537

Sellés, P., Martínez, T., \& Vidal-Abarca, E. (2012). Controversia entremadurezlectoray enseñanzaprecozdela lectura. Revisión histórica y propuestas actuales. Aula Abierta, 40(3), 3-14.

Smith, F. (2012). Understanding reading: A psycholinguistic analysis of reading and learning to read. Londres: Routledge.

Valdivieso, L.B. (2016). El aprendizaje del lenguaje escrito y las ciencias de las lecturas. Un límite entre la psicología cognitiva, las neurociencias y la educación. Límite. Revista Interdisciplinaria de Filosofía y Psicología, 11(36), 50-59.

Wimmer, H., \& Schurz, M. (2010). Dyslexia in Regular Orthographies: Manifestation and a Review on Causation. Dyslexia, 16(4), 283-299. doi: 10.1002/dys.411

Ziegler, J., Bertrand, D., To'th, D., Cse'pe, V., Reis, A., Fai'sca, L., \& Blomert, L. (2010). Orthographic depth and its impact on universal predictors of reading: A cross-language investigation. Psychological Science, 21(4), 551-559. doi: $10.1177 / 0956797610363406$ 